\title{
Fontes de informação sobre benefícios à prática de atividade física e fatores associados em adolescentes: estudo de base escolar
}

\author{
Sources of information about physical activity benefits and associated \\ factors in adolescents: school-based study
}

Marcelo Cozzensa da Silva', Patricia Becker Engers', Guilherme Fonseca Vilela', Carla Franciela Spohr, Airton José Rombaldi

\section{Resumo}

O objetivo do estudo foi verificar as fontes de informação sobre a importância da prática de atividade física e sua associação com nível de atividade física em estudantes do ensino médio da zona urbana da cidade de Pelotas, RS. Foi conduzido um estudo transversal de base escolar na cidade de Pelotas, com uma amostra de 1233 alunos de 13 a 19 anos. Foi autoaplicado um questionário contendo variáveis sociodemográficas, econômica, comportamentais e de saúde. A fonte de informação sobre importância da prática foi avaliada em duas etapas, a primeira pela pergunta: "você já foi informado que a atividade física faz bem para a saúde?” e, em caso positivo, pela solicitação de marcar a(s) opção(ões) de onde veio a informação. A prática de atividade física no lazer foi avaliada por meio de questionário. As análises bruta e ajustada foram realizadas por meio de Regressão de Poisson. Quase a totalidade dos alunos $(99,8 \%)$ relatou ter sido informado que a atividade física faz bem a saúde. Entre as fontes de informação mais relatadas para rapazes e moças, respectivamente, encontram-se a televisão (93,4\% IC $_{95 \%} 91,1-95,7$; $\left.95,9 \% \mathrm{IC}_{95 \%} 91,0-95,3\right)$, professor de educação física $\left(85,6 \% \mathrm{IC}_{95 \%}\right.$ $\left.81,1-86,8 ; 84,1 \% \mathrm{IC}_{95 \%} 82,4-86,8\right)$ e médico $\left(75,9 \% \mathrm{IC}_{95 \%} 72,2-79,4\right.$; $\left.73,3 \% \mathrm{IC}_{95 \%} 69,8-76,7\right)$, não havendo diferença estatística entre os sexos. Após ajuste para fatores de confusão, somente a fonte de informação oriunda dos amigos permaneceu associada à prática de atividade física. Aqueles que receberam informação dos amigos mostraram $41,0 \%$ mais probabilidade de realizar atividades físicas no lazer quando comparados àqueles que não receberam.

\section{Palavras-chave}

Acesso a informação; Atividade motora; Adolescentes; Saúde; Epidemiologia.

\begin{abstract}
The aim of the study was to investigate the sources of information about the importance of physical activity and its association with physical activity level among high school students from the urban area of Pelotas, Brazil. A school-based study was conducted in Pelotas, with a sample of 1233 students 13-19 years-old. A self-administered questionnaire containing sociodemographic, economic, behavioral and health variable was applied. The source of information on importance of practice was assessed in two stages, the first by the question, "Have you been informed that physical activity is good for health?" and, if so, by request mark (s) option (s) where the information was obtained. The practice of leisure time physical activity was assessed through a questionnaire. The crude and adjusted analysis were conducted by Poisson regression. Almost all students (99.8\%) reported having been informed that physical activity is good for health. Among the most frequently reported sources of information for men and women, respectively, were television (93.4\% - 95\% CI=91.1 to 95.7; 95.9\% - 95\% CI=91.0 to 95.3), physical education teacher $(85.6 \%-95 \% C I=81.1$ to $86.8 ; 84.1 \%$ -95\% CI $=82.4$ to 86.8$)$ and physician (75.9\% - 95\% CI= 72.2 to 79.4; 73.3\% 95\% - CI= 69.8 to 76.7), with no statistical difference between the sexes. After adjustment for confounders, friends were the only source of information associated with the practice of physical activity. Those who received information from friends showed 41.0\% more likely to engage in physical activity during leisure time compared to those who did not.
\end{abstract}

\section{Keywords}

Access to Information; Motor Activity; Adolescents; Health; Epidemiology.

\section{Introdução}

A prática de atividades físicas como instrumento da

1 Universidade Federal de Pelotas, Programa de Pós-Graduação em Educação Física, Pelotas, Rio Grande do Sul, Brasil. melhora da saúde e qualidade de vida de indivíduos de todas as idades se encontra fortemente solidificada na literatura ${ }^{1}$. Especificamente na adolescência, existem evidências de que a atividade física proporciona benefícios à saúde óssea, contribui para a me- 
lhora do perfil lipídico e metabólico e redução do percentual de gordura corporal. Além disso, a exposição à atividade física na adolescência parece ser um preditor de práticas na idade adulta ${ }^{2-4}$.

O nível de conhecimento de um indivíduo está estreitamente ligado às informações que recebeu em relação a um determinado tema ${ }^{5}$. Partindo desse raciocínio, Nahas ${ }^{6}$ relata que pesquisas em diversas áreas têm mostrado que o conhecimento sobre um determinado tema está relacionado com a atitude que uma pessoa pode tomar diante dessa questão. Segundo o mesmo autor, pessoas com um maior conhecimento sobre os benefícios da prática de atividades físicas, normalmente, são mais ativas fisicamente.

Apesar da literatura demostrar importantes evidências dos benefícios da prática de atividades físicas para a saúde dos adolescentes, tais informações não chegam aos jovens diretamente por essa fonte. Tal faixa de idade parece receber tais informações via meios de comunicação, profissionais da área de saúde, familiares e amigos. Estudo de Mendes e colaboradores afirma que pouco se sabe sobre o impacto dessas fontes de informação sobre a prática de atividade física na população ${ }^{5}$.

Domingues et $\mathrm{al}^{7}$ e Knuth et $\mathrm{al} .{ }^{8}$ mostraram em seus estudos que o conhecimento sobre os benefícios da prática de atividade física não conduz, necessariamente, a mudanças no estilo de vida dos indivíduos. Entretanto, os autores complementam que sua ausência inviabiliza as transformações de padrão comportamental. Nesse sentido, inferindo-se que as informações sobre os benefícios da atividade física advêm de fontes diversas, ainda não se tem informações do impacto da cada fonte na prática efetiva de atividades físicas por esses indivíduos. Partindo dessa premissa, o estudo buscou verificar as fontes de informação sobre a importância da prática de atividade física e sua associação com nível de atividade física em estudantes do ensino médio da zona urbana da cidade de Pelotas, RS.

\section{Métodos}

Foi conduzido um estudo do tipo transversal de base escolar na cidade de Pelotas, no ano de 2009. Essa cidade está localizada no extremo sul do Rio Grande do Sul e possui cerca de 340.000 habitantes. A pesquisa foi desenvolvida em conjunto por três mestrandos do Programa de Pós-graduação em Educação Física da Universidade Federal de Pelotas.

O tamanho da amostra foi calculado através do programa Epi Info, versão 6.02. O cálculo foi realizado mais de uma vez, de modo que o maior tamanho de amostra necessária ao estudo foi determinado. Como essa pesquisa fez parte de um consórcio, o número de indivíduos que compôs a amostra final foi aquele necessário para o pesquisador que necessitou da maior amostra para seu estudo. Para determinação da prevalência, o cálculo de tamanho amostral considerou frequência de conhecimento sobre atividade física de 25\%, erro aceitável de 3,0 pontos percentuais, nível de confiança de $95 \%$. O número de sujeitos para compor a amostra foi de 800 estudantes; com o acréscimo para perdas e recusas de mais $10 \%$ e de 1,5 para efeito de delineamento, a amostra necessária foi de 1320 indivíduos. Para o estudo de associação entre o desfecho e as variáveis independentes, considerou-se um nível de confiança de $95 \%$, um poder de $80 \%$, odds de exposição (nível socioeconômico $=1 / 9$ ), risco relativo de 1,8 e prevalência estimada nos não expostos de $20 \%$, o número de indivíduos calculado foi de 700 ; acrescidos $10 \%$ perdas e recusas, $15 \%$ para controle de fatores de confusão e 1,5 para efeito de delineamento, o tamanho amostral necessário resultou em 1328 pessoas. O maior cálculo amostral dos estudos integrantes do consórcio foi de 1350 alunos. 
A logística do trabalho teve início com a verificação do número de escolas urbanas que possuíam ensino médio na cidade de Pelotas através de informações obtidas junto a $5^{\text {a }}$ Coordenadoria de Educação (rede pública estadual), Secretaria Municipal de Educação (rede pública municipal) e diretamente nas escolas públicas federais e escolas particulares do município. Posteriormente, todas as instituições escolares foram visitadas para que preenchessem um formulário explicitando o número total de alunos e totais por turma, turno e série. Fizeram parte do processo de seleção da amostra 24 escolas, sendo 15 unidades estaduais (5680 alunos, representando $62 \%$ da população total) seis particulares (1123 alunos, representando $12 \%$ da população total; duas federais (1570 alunos, representando 17\% da população total) e uma municipal (860 alunos, representando 9\% da população total), totalizando 9233 alunos matriculados. Uma escola particular recusou-se a participar do estudo. Através dessas informações procedeu-se a seleção das escolas, a qual foi realizada em um processo em múltiplos estágios. Primeiramente, todas as escolas foram estratificadas segundo a rede de ensino (escolas públicas - redes federal, estadual, municipal - e particulares) e, dentro dessa, proporcional ao tamanho - número de alunos).

Uma solicitação de autorização contendo informações gerais sobre a pesquisa foi entregue aos órgãos gestores das escolas sorteadas para a realização da mesma. Após o sorteio das turmas que compuseram a amostra, foi realizada uma breve explanação sobre a pesquisa aos alunos. Cada aluno, menor de 18 anos, recebeu uma cópia do "termo de consentimento livre e esclarecido" para participar do estudo, o qual foi assinado pelos pais ou responsáveis e devolvido antes do dia da aplicação do questionário.

O questionário aplicado foi testado a alunos de três turmas de uma escola de ensino médio ( $\mathrm{n}=76)$ não sorteada para compor a amostra com o objetivo de avaliar o entendimento das questões pelos alunos, monitorar o tempo utilizado para responder o questionário, servir como treinamento final para os pesquisadores e aperfeiçoar o instrumento tornando-o mais adequado à compreensão da amostra estudada.

Os questionários foram auto-aplicados em sala de aula na presença dos pesquisadores, no período de junho a setembro de 2009. Duas profissionais da área de nutrição coletaram as informações relacionadas a massa corporal (estudantes usando roupas leves e descalços em uma balança digital marca SOEHNLE 7755, com capacidade de $150 \mathrm{~kg}$ e precisão de $100 \mathrm{~g}$ ) e estatura (estadiômetro tipo trena com $200 \mathrm{~cm}$ e precisão de $0,1 \mathrm{~cm}$ ), ambos rotineiramente calibrados.

$\mathrm{O}$ instrumento utilizado para a coleta de dados continha 101 perguntas sobre características socioeconômicas, demográficas, comportamentais, nutricional e de saúde, além de questões específicas de conhecimento sobre atividades físicas. $O$ objetivo das questões sobre conhecimento foi verificar os benefícios da atividade física para a saúde, para a prevenção de doenças crônicas, finalidades da atividade física e efeitos da mesma no organismo humano.

As variáveis independentes analisadas foram: sexo (masculino e feminino), cor da pele (auto-referenciada e classificada como branca e não branca), idade (em anos completos), nível socioeconômico (conforme classificação da ABEP) ${ }^{9}$ e escolaridade $\left(1^{\mathrm{a}}, 2^{\mathrm{a}}\right.$ e $3^{\mathrm{a}}$ série do ensino médio). As variáveis comportamentais medidas foram tabagismo (fumante atual, não fumante e ex-fumante) e nível de atividade física (referenciada nos últimos sete dias, sem contar as aulas de educação física). A variável de saúde foi caracterizada pela autopercepção de saúde (excelente, muito boa, boa, ruim e péssima) e a variável nutricional pelo índice de massa corporal (Cole et al. $)^{10}$. A fonte de informação sobre a atividade física como meio de benefício à saúde foi realizada através de duas perguntas. A primeira foi "você já foi informado que a atividade física faz bem para a saúde?” e, em caso positivo, era solicitado ao adoles- 
cente marcar a(s) opção(ões) (múltipla escolha) de onde foi oriunda a informação: médico, televisão, jornal, revista, professor de educação física, professor de outras disciplinas, amigos, parentes e outras fontes. O desfecho, prática de atividade física no lazer, foi coletada com base no instrumento elaborado por Bastos et al..$^{11}$ o qual mede (sem contar as práticas executadas durante a disciplina de educação física escolar), as atividades físicas realizadas, o número de dias e horas por dia praticadas. Foi definido como insuficientemente ativo no lazer os adolescentes que realizaram menos de 300 minutos de atividade física moderada a vigorosa por semana ${ }^{12}$.

Os dados foram duplamente digitados utilizando o programa Epi Info 6.0, com checagem automática de consistência, para correção de possíveis erros. A análise descritiva caracterizou a amostra total, conforme variáveis demográficas, socioeconômicas, comportamentais, de saúde e nutricional. A análise dos dados foi realizada no programa estatístico Stata 10.0. As análises bruta e ajustada foram realizadas através de regressão de Poisson sendo o desfecho (nível de atividade física) ajustado para as variáveis independentes em estudo, sendo a principal exposição as fontes de informação sobre a atividade física. Para a realização da análise foi levada em consideração a hierarquia de determinação da prática de atividade física seguindo modelo utilizado para outros desfechos em saúde preconizado por Victora et al. ${ }^{13}$. O modelo proposto para a hierarquia citada foi constituído de cinco níveis: o primeiro em que estavam inseridas as variáveis demográficas, o segundo em que estavam as variáveis socioeconômicas, o terceiro que no qual esteve a série de estudo dos adolescentes, o quarto que abrangeu as variáveis comportamentais e de saúde, no quinto onde esteve contido as fontes de informação sobre a atividade física. Os efeitos das variáveis foram controlados para as mesmas que se encontravam em seu nível hierárquico e para aquelas em nível superior.

O projeto foi aprovado pelo Comitê de Ética em Pesquisa da Escola Superior de Educação Física da Universidade Federal de Pelotas sob número 031/2009.

\section{Resultados}

Foram estudados 1233 alunos de 13 a 19 anos matriculados no ensino médio da rede de ensino da cidade de Pelotas/RS/, já descontadas a percentagem de 8,7\% de perdas e recusas.

A média de idade foi de 15,9 anos ( $\mathrm{DP}=1,14$ anos), sendo que $54 \%$ eram do sexo feminino e $4 / 5$ possuíam cor de pele branca. A maioria dos estudantes encontrava-se nos níveis econômico B e C (91,1\%).

Quanto à auto-percepção de saúde, mais de 90\% dos adolescentes relataram sua saúde de boa a excelente e cerca de $1 / 4$ da amostra encontrava-se nas categorias referente a sobrepeso/obesidade segundo o índice de massa corporal. A prevalência total de indivíduos insuficientemente ativos no lazer foi de $63,9 \%$ sendo (75,7\% entre as meninas e $50,1 \%$ entre os rapazes) (Tabela 1 ).

Quase a totalidade dos alunos (99,8\%) relatou ter sido informado que a atividade física faz bem a saúde. A figura 1 mostra as fontes de informação sobre a relação positiva entre prática de atividade física e saúde dos adolescentes escolares de acordo com o sexo dos indivíduos. Entre as fontes de informação mais relatadas para rapazes e moças, respectivamente, encontram-se a televisão $\left(93,4 \% \mathrm{IC}_{95 \%} 91,1\right.$ 95,7; 95,9\% $\left.\mathrm{IC}_{95 \%} 91,0-95,3\right)$, professor de educação física $\left(85,6 \% \mathrm{IC}_{95 \%} 81,1-86,8\right.$; $\left.84,1 \% \mathrm{IC}_{95 \%} 82,4-86,8\right)$ e médico $\left(75,9 \% \mathrm{IC}_{95 \%} 72,2-79,4 ; 73,3 \% \mathrm{IC}_{95 \%} 69,8-76,7\right)$, não havendo diferença estatística entre os sexos para cada uma das fontes mencionadas. Por outro lado, houve diferença estatística para as fontes revista $(\mathrm{p}<0,001)$, amigos $(\mathrm{p}=0,002)$ e professores de outras disciplinas $(\mathrm{p}=0,04)$ (Figura 1). Quando 
TABELA 1 - Descrição da amostra de escolares adolescentes cursando ensino médio nas escolas da cidade de Pelotas/RS/Brasil.

\begin{tabular}{|c|c|c|}
\hline \multirow[t]{2}{*}{ Variável } & \multicolumn{2}{|c|}{ Total } \\
\hline & $n$ & $\%$ \\
\hline \multicolumn{3}{|l|}{$\operatorname{Sexo}(n=1233)$} \\
\hline Masculino & 567 & 46,0 \\
\hline Feminino & 666 & 54,0 \\
\hline \multicolumn{3}{|c|}{ Idade (anos completos) ( $n=1233$ ) } \\
\hline 14 anos & 156 & 12,7 \\
\hline 15 anos & 277 & 22,5 \\
\hline 16 anos & 401 & 32,5 \\
\hline 17 anos & 291 & 23,6 \\
\hline 18/19 anos & 108 & 8,7 \\
\hline \multicolumn{3}{|l|}{ Cor da pele $(n=1201)$} \\
\hline Branco & 949 & 79,0 \\
\hline Não branco & 252 & 21,0 \\
\hline \multicolumn{3}{|l|}{ Nivel econômico (n=1036) } \\
\hline A & 82 & 7,9 \\
\hline B & 563 & 54,3 \\
\hline$C / D$ & 368 & 37,8 \\
\hline \multicolumn{3}{|l|}{ Escolaridade $(n=1233)$} \\
\hline 10 ano ensino médio & 470 & 38,1 \\
\hline $2^{\circ}$ ano ensino médio & 397 & 32,2 \\
\hline $3^{\circ}$ ano ensino médio & 366 & 29,7 \\
\hline \multicolumn{3}{|c|}{ Nível de atividade física ( $n=1233$ ) } \\
\hline Insuficientemente ativos & 788 & 63,9 \\
\hline Ativos & 445 & 36,1 \\
\hline \multicolumn{3}{|c|}{ Índice de Massa Corporal $(n=1191)$} \\
\hline Normal & 889 & 74,7 \\
\hline Sobrepeso & 235 & 19,7 \\
\hline Obesidade & 67 & 5,6 \\
\hline \multicolumn{3}{|l|}{ Tabagismo $(n=1221)$} \\
\hline Não fumante & 1150 & 94,2 \\
\hline Fumante & 71 & 5,8 \\
\hline \multicolumn{3}{|c|}{ Autopercepção de saúde (n=1233) } \\
\hline Excelente & 233 & 18,9 \\
\hline Muito boa & 460 & 37,3 \\
\hline Boa & 430 & 34,9 \\
\hline Regular & 104 & 8,4 \\
\hline
\end{tabular}

considerada as fontes oriundas exclusivamente do ambiente escolar, as prevalências de informação sobre a associação positiva entre atividade física e saúde, houve diferença estatisticamente significante entre os professores de educação física $(84,8 \%)$ em relação aos professores das demais disciplinas $(51,0 \%)(\mathrm{p}<0,001)$.

A tabela 2 descreve a análise da relação entre fontes de informação e prática de atividades físicas entre os adolescentes escolares. Após ajuste para fatores de confusão, somente a fonte de informação oriunda dos amigos permaneceu associada ao desfecho. Aqueles que receberam informação dos amigos mostraram $41 \%$ mais probabilidade de realizar atividades físicas no lazer quando comparados aqueles que não receberam $\left(\mathrm{RP}=1,41 ; \mathrm{IC}_{95 \%} 1,15-1,71\right)$. 


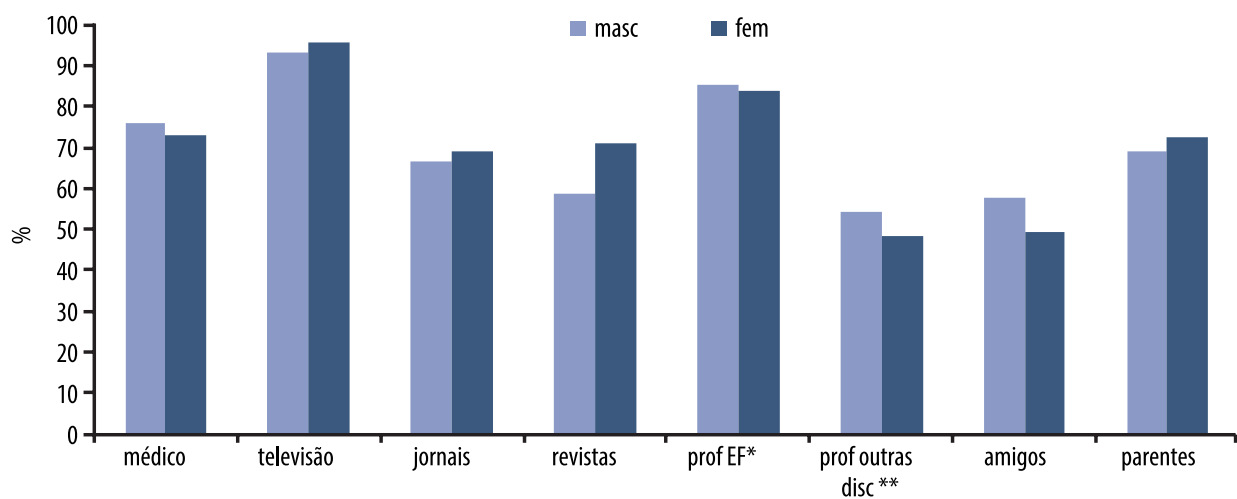

FIGURA 1 - Fontes de informação sobre a relação positiva entre prática de atividade física e saúde de adolescentes escolares da cidade de Pelotas/RS de acordo com o sexo ( $n=1229)$.

* prof $E F=$ professor de educação física; prof outras disc= professor de outras disciplinas; valor $p$ médico $=0,3$; valor $p$ televisão $=0,05$; valor $p$ jornal $=0,4$; Valor $p$ revistas $=<0,001$; Valor $p$ professor de educação física $=0,5$; Valor $p$ outras disciplinas $=0,04$; Valor $p$ amigos $=0,002$; Valor $p$ parentes $=0,2$.

TABELA 2 - Associação entre fonte de informação e prática de atividades físicas entre adolescentes do ensino médio da cidade de Pelotas.

\begin{tabular}{lccc}
\hline Fontes de informação & Bruta (IC95\%) & Ajustada* (IC95\%) & P \\
\hline Médico & $1,21(1,00,1,45)$ & $1,10(0,90,1,26)$ & 0,300 \\
\hline Televisão & $0,88(0,65,1,20)$ & $0,98(0,66,1,46)$ & 0,900 \\
\hline Jornais & $0,96(0,82,1,12)$ & $0,93(0,76,1,13)$ & 0,500 \\
\hline Revistas & $0,91(0,79,1,07)$ & $0,95(0,78,1,16)$ & 0,600 \\
\hline Professor de Educação Física & $1,14(0,92,1,43)$ & $1,08(0,83,1,39)$ & 0,600 \\
\hline Professor de outras disciplinas & $1,07(0,92,1,24)$ & $0,86(0,72,1,03)$ & 0,100 \\
\hline Amigos & $1,40(1,20,1,64)$ & $1,41(1,15,1,71)$ & 0,001 \\
\hline Parentes & $1,12(0,94,1,32)$ & $0,93(0,76,1,15)$ & 0,500 \\
\hline
\end{tabular}

*Ajustada para as variáveis dos níveis superiores e do mesmo nível hierárquico: 10 nível: sexo, cor da pele; $2^{\circ}$ nível: nível econômico 30: série de estudo; 4 º́vel: índice de massa corporal, fumo; $5^{\circ}$ nível: fontes de informação.

\section{Discussão}

Quase a totalidade dos alunos relatou ter sido informado que a atividade física faz bem a saúde. Os dados encontrados na literatura mostram resultados diversos a respeito do tema. Estudo de base populacional realizado na cidade de Pelotas identificou alta prevalência de informação recebida sobre importância da prática de atividade física entre adultos $(76,1 \%)^{5}$. Entretanto, outro estudo de base populacional realizado nas regiões sul e nordeste do Brasil encontrou prevalência de aconselhamento de prática de atividade física de $27,7 \%{ }^{14}$. Estudo recente realizado em 100 municípios do Brasil mostrou uma prevalência de 30\% de aconselhamento para prática de atividade física ${ }^{15}$. Prevalência mais próxima daquela encontrada no presente estudo foi a relatada por Mendes e colaboradores 5 . Diferente dos estudos que foram desenvolvidos em unidades básicas de saúde ou próximo a elas, os quais consideraram como fonte de informação somente as originadas pelos profissionais de saúde, o presente estudo e o de Mendes e colaboradores 5 , incluíram, além das informações fornecidas pelo médico, outras fontes, tais como televisão e professor.

As fontes mais frequentes de informação sobre atividade física relacionada à saúde foram oriundas da televisão, professor de educação física, parentes e médicos. Estudo realizado no mesmo município, com indivíduos de faixa etária diferente do presente estudo, também encontrou a televisão como a maior fonte de informação, tanto para homens $(29,9 \%)$, quanto para as mulheres $(25,9 \%)^{5}$. Estudo com profissionais de saúde do município de São Caetano (SP) identificou os pro- 
gramas de televisão $(84,1 \%)$ e os artigos científicos $(83,3 \%)$ como fontes de informação mais frequentes entre aqueles profissionais que recomendavam a atividade física ${ }^{16}$. A Organização Mundial da Saúde salienta que a televisão, o rádio e outras mídias de entretenimento alcançam um elevado percentual dos adolescentes das zonas urbanas, e têm potencialidades para chegar até as zonas rurais, ajudando na disseminação da importância da prática de atividades físicas para a saúde destes indivíduos ${ }^{17}$. Apesar disso, no presente estudo, a mídia televisiva não foi capaz de modificar os hábitos de prática desses adolescentes. É necessário reavaliar o real impacto da informação transmitida através deste meio de comunicação nas mudanças de comportamento da população, visto que a mesma atinge milhares de pessoas nas diferentes faixas de idade e de distintos níveis econômicos ${ }^{18,19}$.

A prevalência de informação recebida através do professor de educação física foi elevada. Esse profissional vem sendo descrito como uma das fontes mais frequentes de informação sobre o tema em diferentes faixas etárias, até mesmo entre indivíduos adultos, os quais, em pequena parcela, frequentam espaços em que o professor de educação física esteja atuando ${ }^{5,20}$. Estudo de coorte realizada na cidade de Pelotas-RS verificou que 99,1\% dos adolescentes da zona urbana matriculados nas escolas participavam das aulas de educação física ${ }^{21}$. Tal fato reforça a importância do papel do professor de educação física na promoção da atividade física no ambiente escolar, incumbindo ao mesmo o fornecimento de informações acerca da importância da prática da atividade para a saúde, bem como da oferta de atividades orientadas e ações educativas.

Os médicos, como uma das principais fontes de informação sobre atividade física relacionada à saúde, foram bastante lembrados entre os sujeitos do estudo. Domingues et al. ${ }^{7}$ relataram em estudo de base populacional realizado com adultos da cidade de Pelotas, que os médicos foram considerados a segunda maior fonte de informação sobre os benefícios da atividade física, ficando atrás somente dos meios de comunicação. Estudo de Eden et al. ${ }^{22}$ visando determinar se o aconselhamento médico realizado nos serviços de atenção primária melhora os níveis de atividade física da população atendida, não demonstrou evidências suficientes capaz de suportar tal afirmação.

No presente estudo, após a análise ajustada, apenas a fonte de informação amigos permaneceu associada ao desfecho. Vários estudos apontam para a relação do apoio social dos amigos e também da família para a prática de atividade física dos adolescentes ${ }^{23-26}$. Segundo Beets et al. ${ }^{27}$, tal apoio social pode ser dado através de incentivo verbal, acompanhamento da prática e transporte dos adolescentes até o local de onde a atividade será realizada.

Cheng e colaboradores ${ }^{23}$, ao analisarem a influência do apoio social dos pais e amigos no nível de atividade física dos adolescentes da cidade de João Pessoa- PB verificaram uma forte associação entre os mesmos. Resultado similar foi reportado no estudo de Fermino e colaboradores ${ }^{24}$ com escolares da rede pública de Curitiba- PR. Outro estudo ${ }^{25}$ realizado com adolescentes do nordeste do Brasil também demonstrou que aqueles que receberam apoio social dos pais e dos amigos apresentaram, respectivamente, mais chance de serem ativos (sexo masculino: respectivos apoios sociais de pais e amigos: $\mathrm{RO}=1,54\left(\mathrm{IC}_{95 \%}=1,06\right.$ a 2,24) e $\mathrm{RO}=$ $2,94\left(\mathrm{IC}_{95 \%}=1,96\right.$ a 4,39); sexo feminino: respectivos apoios sociais de pais e amigos: $\mathrm{RO}=1,46\left(\mathrm{IC}_{95 \%=} 1,08\right.$ a 1,98) e $\mathrm{RO}=2,65\left(\mathrm{IC}_{95 \%=} 1,94\right.$ a 3,61). Prado e colabora dores $^{26}$ verificaram que a companhia frequente da família e dos amigos foi o apoio social mais importante para a prática de atividade física dos adolescentes sendo que o apoio dos amigos foi mais relevante. Neste sentido, percebe-se que nesta fase 
da vida a influência dos amigos tende ser mais importante do que a dos pais, pois com o aumento da idade os jovens passam a praticar mais atividade física com os amigos do que com a família ${ }^{28}$. Segundo Gonçalves et al. ${ }^{29}$, o contato com amigos fora do ambiente escolar pode levar o indivíduo a ser mais ativo.

Algumas limitações do estudo devem ser descritas. A causalidade reversa, que pode dificultar a determinação temporal da relação entre causa e efeito, é uma das limitações mais comuns entre estudos de delineamento transversal. Em nosso estudo, esse viés pode estar presente na relação entre fonte de informação e prática. Outro ponto a ser salientado é que não foram coletados, na época de realização do estudo, dados sobre fontes de informação oriundas da internet, mídia na qual existe grande quantidade dessas informações. Segundo Cassidy e $\mathrm{Baker}^{30}$, muitas pessoas usam cada vez mais a internet para pesquisar questões relacionadas com a saúde, apesar de o conteúdo da Internet, ao contrário de outras formas de mídia, não ser regulamentado. Pontos positivos devem ser ressaltados, em especial o cuidado metodológico do estudo e a amostra representativa de escolares do município de Pelotas.

Concluiu-se que quase a totalidade dos entrevistados recebeu informação relacionada aos benefícios da prática de atividade física relacionado à saúde, sendo, ainda, a televisão a fonte mais lembrada pelos indivíduos, superando os profissionais da área da saúde. Apesar disso, somente a fonte de informação amigos esteve associada efetivamente a prática dos adolescentes. Baseado nessas informações, iniciativas que estimulem a prática física em grupos de adolescentes podem ser uma grande alternativa para a difusão e continuidade da mesma entre esses indivíduos. Nesse contexto, a escola reúne todas as ferramentas para o sucesso, pois nela se encontram quase a totalidade dos indivíduos nessa faixa etária, com grande relacionamento social entre eles e expostos a mais competente fonte de informação sobre os benefícios da prática: os professores de educação física. Sugere-se a realização de novos estudos referentes a essa temática, a qual, apesar de muito importante, ainda necessita de maior sustentação científica de sua associação.

\section{Contribuiç̧ão dos autores}

M.C. Silva e A.J.Rombaldi participaram da criação do projeto, análise dos dados e todas as fases de redação do artigo. P.C. Engers, G.F. Vilela, C.F. Spohr supervisionaram o trabalho de campo, realizaram a tabulação dos dados, revisão de literatura e participaram da redação final do artigo. Os autores aprovaram a versão final do manuscrito.

\section{Referências}

1. Lee IM, Shiroma EJ, Lobelo F, Puska P, Blair SN, Katzmarzyk PT. Effect of physical inactivity on major non-communicable diseases worldwide: an analysis of burden of disease and life expectancy. Lancet. 2012; 380:219-229.

2. Hallal PC, Victora CG, Azevedo MR, Wells JC. Adolescent physical activity and health: a systematic review. Sports Med. 2006; 36:1019-30.

3. Lazzoli JK, Nóbrega ACL, Carvalho T, Oliveira MAB, Teixeira JAC, Leitão MB, et al. Atividade física e saúde na infância e adolescência. Rev Bras Med Esporte. 1998; 4: 107-09.

4. Azevedo MR, Araujo CL, Cozzensa da Silva M, Hallal PC. Tracking of physical activity from adolescence to adulthood: a population-based study. Rev Saude Publica. 2007; 41(1):69-75.

5. Mendes MA, Rombaldi AJ, Azevedo MR, Bielemann RM, Hallal PC. Fontes de informação sobre a importância da atividade física: estudo de base populacional. Rev Bras Ativ Fis Saude. 2010; 15(3): 163-69.

6. Nahas M. Atividade Física, Saúde e qualidade de vida: Conceitos e sugestões para um estilo de vida ativo. Londrina: Midiograf, 2010.

7. Domingues MR, Araujo CL, Gigante DP. Knowledge and perceptions of physical exercise in an adult urban population in Southern Brazil. Cad Saude Publica. 2004; 20(1):204-15.

8. Knuth AG, Bielemann RM, SilvaSG, Borges TT, Del Duca GF, KremerMM, etal. Publicknowledge on the role of physical activity in the prevention and treatment of diabetes and hypertension: a population-based study in southern Brazil. Cad Saude Publica. 2009; 25(3): 513-20. 
9. Associação Brasileira de Empresas de Pesquisa (ABEP). Critério de classificação econômica Brasil.2009. Disponível em: http://www.abep.org. Acesso em: 25 nov 2015.

10. Cole TJ, Bellizzi MC, Flegal KM, Dietz WH. Establishing a standard definition for child overweight and obesity worldwide: international survey. Br Med J. 2000; 320: 1-6.

11. Bastos JP, Araújo CLP, Hallal PC. Prevalence of insufficient physical activity and associated factors in brazilian adolescents. J Phys Act Health. 2008; 5:777-794.

12. Biddle S, Cavill N, Sallis J. Young and active? Young people and health-enhancing physical activity evidence and implications. London: Health Educ Authority, 1998.

13. Victora CG, Huttly SR, Fuchs SC, Olinto MT. The role of conceptual frameworks in epidemiological analysis: a hierarchical approach. Int J Epidemiol. 1997; 26: 224-227.

14. Siqueira FV, Nahas MV, Facchini LA, Silveira DS. Counseling for physical activity as a health education strategy. Cad Saude Publica. 2009; 25(1): 203-13.

15. Duro SM, Tomasi E, Siqueira FV, Silveira DS, Thumé E, Facchini LA. Adult physical activity counseling by health professionals in Brazil: A national urban population survey. J Phys Act Health. 2015; 12(8): 1177-1183.

16. Andrade DR. Atividade física e promoção da saúde: conhecimento e prática dos profissionais de saúde das unidades básicas de saúde do município de São Caetano do Sul - São Paulo. Dissertação de Mestrado. São Paulo: Faculdade de Saúde Pública da Universidade de São Paulo; 2001.

17. WHO (2001). Saúde dos adolescentes: Estratégia para a região africana. WHO Regional Office for Africa. Brazzaville

18. Proctor MH, Moore LL, Gao D, Cupples LA. Television viewing and change in body fat from preschool to early adolescence: The Framingham Children's Study. Int J Obes Relat Metab Disord. 2003; 27: 827-33.

19. Hancox R, Milne B, Poulton R. Association between child and adolescent television viewing and adult health: a longitudinal birth cohort study. Lancet. 2004; 364:257-62.

20. Silva SM, Facchini LA, Thumé E, Tomasi E. Physical Activity in Adults: Counseling by Health Workers and Behavioral Change. [cited]; Available from: http://epidemioufpel. org.br/_teses_e_dissertacoes/dissertacoes2.php?id_dissertacoes=115.

21. Hallal PC, Bertoldi AD, Gonçalves H, Victora CG. Prevalence of sedentary lifestyle and associated factors in adolescents 10 to 12 years of age. Cad Saude Publica. 2006; 6(22): 1277-87.

22. Eden KB, Orleans CT, Mulrow CD, Pender NJ, Teutsch SM. Does Counseling by Clinicians Improve Physical Activity? A Summary of the Evidence for the U.S. Preventive Services Task Force. Ann Intern Med. 2002; 137(3): 208-15.

23. Cheng LA, Mendonça G, Farias Júnior JC. Physical activity in adolescents: analysis of social influence of parents and friends. J Pediatr. 2014; 90(1): 35-41.

24. Fermino RC, Rech CR, Hino AAF, Añez CRR, Reis RS. Atividade física e fatores associados em adolescentes do ensino médio de Curitiba, Brasil. Rev Saude Publica. 2010; 44(6): 986-95.

25. Farias Júnior JC, Reis RS, Hallal PC. Physical activity, psychosocial and perceived environmental factors in adolescents from Northeast Brazil. Cad Saude Publica. 2014; 30 (5): 941-951.

26. Prado CV, Lima AV, Fermino RC, Añez CRR, Reis RS. Apoio social e prática de atividade física em adolescentes da rede pública de ensino: qual a importância da família e dos amigos? Cad Saude Publica. 2014; 30 (4): 827-838.

27. Beets MW, Cardinal BJ, Alderman BL. Parental social support and the physical activityrelated beh aviors of youth: a review. Health Educ Behav. 2012;37:621-44.

28. Kirby J, Levin KA, Inchley J. Parental and peer influences on physical activity among scottish adolescents: a longitudinal study. J Phys Act Health 2011; 8:785 -93.

29. Gonçalves H, Hallal PC, Amorim TC, Araújo CLP, Menezes AMB. Fatores socioculturais e nível de atividade física no início da adolescência. Rev Panam Salud Publica. 2007; 22(4):246-53.

30. Cassidy JT, Baker JF. Orthopaedic Patient Information on the World Wide Web: An Essential Review. J Bone Joint Surg Am. 2016; 17;98(4):325-38.

ENDEREÇO PARA CORRESPONDÊNCIA MARCELO COZZENSA DA SILVA cozzensa@terra.com.br
PPG em Educação Física, Universidade Federal de Pelotas. Rua Luis de Camões 625 - CEP: 96055-630. one (fax): (53) 3273-3851. 